\title{
THE RANDOM EIGENVALUE PROBLEM FOR A DIFFERENTIAL EQUATION CONTAINING SMALL WHITE NOISE*
}

\author{
BY \\ NING-MAO XIA \\ East China Institute of Chemical Technology, Shanghai, China
}

Abstract. By means of the extended Ito integral and the shooting method, this paper concerns the random problem containing white noise $B(t, \omega)$ :

$$
\left[-p(t) u^{\prime}(t)\right]^{\prime}+[q(t)+\varepsilon B(t, \omega)] u=\lambda u, \quad u(0)=0, \quad u(1)=0 .
$$

When $\varepsilon$ is small, the existence and asymptotic expansions for the solutions can be obtained, and the normal properties for the first correction terms can be proved. Formulas for evaluation are derived and one example of the Schrödinger equation is given to illustrate the whole procedure.

1. Introduction. The spectral theory for the random operator is of great importance both in theory and applications. When the problem is related to the boundary value problem for ordinary differential equations, many interesting results have been obtained by various means $[1-4,6,10]$. In the case that only a finite number of random variables are contained in the problem, the first-order partial differential equation for the Prüfer functions gives an efficient way to find the density function of the solution [6]. If the sample functions of the random processes in the problem are smooth, then the methods of integral equations, Green's functions, and eigenfunction expansion have been proved to be useful to derive some meaningful properties $[2-4,10]$.

The evaluation of energy levels of a quantum mechanical system leads to the famous Schrödinger equation. In one-dimensional space it often has the form $-u^{\prime \prime}+$ $q(t, \omega) u=\lambda u, u(0)=0, u(1)=0$. When the potential is a homogeneous metrically transitive random field, and almost all of its realizations are bounded below and continuously differentiable, the Feynman-Kac representation of the Green's function has been used to obtain some interesting results [14]. However, for problems involving white noise much less is known, since the necessary measurability for Ito's theory is not always satisfied $[2,7,9]$.

In this paper we will consider the eigenvalue problem containing white noise $B(t, \omega)$ and small parameter $\varepsilon \in\left[0, \varepsilon_{0}\right]$ :

$$
\left[-p(t) u^{\prime}(t)\right]^{\prime}+[q(t)+\varepsilon B(t, \omega)] u=\lambda u, \quad u(0)=0, \quad u(1)=0 .
$$

*Received April 10, 1987. 
As in the deterministic case, if $C^{\prime}$ and $C$ denote the functional spaces with appropriate smoothness, then we always assume that $t \in[0,1], p(t)>0, p(t) \in C^{\prime}$, and $q(t) \in C$. Similar to the initial value problem of the Ito differential equation, if the Wiener process $W(t, \omega)$ and new function $v(t)$ are introduced, then (1.1) can be rewritten in the following form:

$$
\begin{aligned}
d u(t) & =\frac{v(t)}{p(t)} d t, \\
d v(t) & =[q(t)-\lambda] u(t) d t+\varepsilon u(t) d W(t, \omega), \\
u(0) & =0, \\
u(1) & =0 .
\end{aligned}
$$

When $\varepsilon=0$, it reduces to a deterministic problem. By the so-called Prüfer substitution [5] it can be proved to have a discrete spectrum $\lambda_{1}<\lambda_{2}<\cdots<\lambda_{n}<\cdots$, and related functions $u_{1}(t), u_{2}(t), \ldots, u_{n}(t), \ldots ; v_{1}(t), v_{2}(t), \ldots, v_{n}(t), \ldots$ They are deterministic and satisfy the condition:

$$
\int_{0}^{1} u_{i}(t) u_{j}(t) d t=\delta_{i j}, \quad i, j=1,2, \ldots
$$

For $\varepsilon \neq 0$, notice that $(1.2)$ is a random boundary value problem; its solutions (if existent) will depend on $W(t, \omega)$ for $t \in[0,1]$. Hence the $F_{t}$-measurability (which is necessary for the Ito integral) will not be satisfied. To avoid the possible obstacle of measurability, in Sections 2, 3 we will define the extended Ito integral, and then show the existence of the solution for (1.2) by means of series expansions and the shooting method.

When $\varepsilon \ll 1$, these solutions can be expanded in $\varepsilon$ with the form:

$$
\begin{aligned}
\lambda(\omega) & =\lambda_{n}+\varepsilon \lambda^{*}(\omega)+O\left(\varepsilon^{2}\right), \\
u(t, \omega) & =u_{n}(t)+\varepsilon u^{*}(t, \omega)+O\left(\varepsilon^{2}\right), \quad n=1,2, \ldots,
\end{aligned}
$$

where $\lambda^{*}$ is a normal random variable and $u^{*}(t, \omega)$ is a Gaussian process. They have zero mathematical expectations, $\left\langle\lambda^{*}(\omega)\right\rangle=0,\left\langle u^{*}(t, \omega)\right\rangle=0$, and their expressions can be obtained explicitly. All these matters will be discussed and proved in Sec. 4 together with an example of a Schrödinger equation.

2. Definition of the extended Ito integral and solutions of the random initial value problem.

Definition 1. Suppose the following.

(1) For all $t \in[0,1]$, the $\sigma$-algebras of events $F_{t}$ are defined so as to possess the properties:

(a) for $0 \leq t_{1}<t_{2} \leq 1, F_{t_{1}} \subset F_{t_{2}}$,

(b) $W(t, \omega)$ is an $F_{t}$-measurable Wiener process with independent increments,

(c) $W\left(t_{2}, \omega\right)-W\left(t_{1}, \omega\right)$ does not depend on the $\sigma$-algebra $F_{t_{1}}$.

(2) The random process $f(t, \omega)$ is defined for $t \in[0,1]$, and

(a) for every fixed $t \in[0,1], f(t, \omega)$ is an $F_{1}$-measurable function of $\omega$,

(b) it has bounded second moment,

$$
\sup _{t \in[0,1]}\left\langle f^{2}(t, \omega)\right\rangle \leq \text { const },
$$


(c) $f(t, \omega) \in c$, w.p.1 (with probability one).

(3) For fixed $T \in[0,1]$, and any partition of [0,T], $\left(0=t_{0}<t_{1}<\cdots<t_{k}<\cdots<\right.$ $\left.t_{m}=T \leq 1\right)$, the random variable defined by $\sum_{k=0}^{m-1} f\left(t_{k}, \omega\right)\left[W\left(t_{k+1}, \omega\right)-\right.$ $\left.W\left(t_{k}, \omega\right)\right]$ has bounded second moment, and as $\Delta=\max _{k}\left(t_{k+1}-t_{k}\right) \rightarrow 0$, it converges in mean square to a random variable $I(\omega)$,

$$
\lim _{\Delta \rightarrow 0} \sum_{k=0}^{m-1} f\left(t_{k}, \omega\right)\left[W\left(t_{k+1}, \omega\right)-W\left(t_{k}, \omega\right)\right]=I(\omega),
$$

where the limit is independent of the choice of partition, and $\left\langle I^{2}(\omega)\right\rangle<\infty$.

Then $f(t, \omega)$ is said to have the extended Ito integral on $[0, T]$, and is denoted by

$$
\int_{0}^{T} f(t, \omega) d W(t, \omega)=I(\omega)
$$

Remark 1. If we let

$$
H_{2}[0, T]=\left\{\begin{array}{l|l}
f(t, \omega) & \begin{array}{l}
f(t, \omega) \in C[0, T] \text { w.p. } 1, \int_{0}^{T}\left\langle f^{2}(t, \omega)\right\rangle d t<\infty \\
f(t, \omega) \text { is } F_{t} \text {-measurable for } t \in[0, T],
\end{array}
\end{array}\right\}
$$

then for any $f(t, \omega) \in H_{2}[0, T]$, the extended Ito integral exists and equals the Ito integral. [See 11, for example.]

Remark 2. Let $a(\omega), b(\omega)$ be $F_{1}$-measurable bounded random variables, and $f_{1}(t, \omega), f_{2}(t, \omega) \in H_{2}[0, T]$. Then the function $a(\omega) f_{1}(t, \omega)+b(\omega) f_{2}(t, \omega)$ has the extended Ito integral, and

$$
\begin{aligned}
\int_{0}^{T}\left[a(\omega) f_{1}(t, \omega)+b\right. & \left.(\omega) f_{2}(t, \omega)\right] d W(t, \omega) \\
& =a(\omega) \int_{0}^{T} f_{1}(t, \omega) d W(t, \omega)+b(\omega) \int_{0}^{T} f_{2}(t, \omega) d W(t, \omega) .
\end{aligned}
$$

The proof can be obtained easily from Definition 1, and for convenience of the statement of Theorem 1, we need the following remark.

Remark 3. For a sequence of random processes $f_{k}(t, \omega) \in H_{2}[0, T], k=0,1, \ldots$, we assume that

(1) there exist three positive constants $A, B, C_{0}$, such that

$$
\begin{gathered}
\left\langle\sup _{t \in[0, T]} f_{k}^{2}(t, \omega)\right\rangle \leq A^{k}(k !)^{2} C_{0}, \\
\left\langle\left[f_{k}\left(t^{\prime}, \omega\right)-f_{k}\left(t^{\prime \prime}, \omega\right)\right]^{2}\right\rangle \leq A^{k}(k !)^{2} B\left|t^{\prime}-t^{\prime \prime}\right|, \quad \text { for } t^{\prime}, t^{\prime \prime} \in[0, T] ;
\end{gathered}
$$

(2) there exist a positive constant $C_{\eta}$ and an $F_{1}$-measurable random variable $\eta(\omega)$ such that $|\eta(\omega)| \leq C_{\eta}<1$, w.p. 1 , and $C_{\eta} A<1$.

Then the series $\sum_{k=0}^{\infty} \frac{\eta^{k}(\omega)}{k !} f_{k}(t, \omega)$ converges uniformly to a random process $f(t, \omega)$ w.p.1, which has continuous sample function and has the extended Ito integral

$$
\int_{0}^{T} f(t, \omega) d W(t, \omega)=\sum_{k=0}^{\infty} \frac{\eta^{k}(\omega)}{k !} \int_{0}^{T} f_{k}(t, \omega) d W(t, \omega) .
$$


Proof. From the assumptions, we know that

$$
\left\langle\sup _{t \in[0, T]}\left[\frac{\eta^{k}(\omega)}{k !} f_{k}(t, \omega)\right]^{2}\right\rangle \leq C_{\eta}^{2 k} \frac{1}{k !^{2}}\left\langle\sup _{t \in[0, T]} f_{k}^{2}(t, \omega)\right\rangle \leq\left(C_{\eta}^{2} A\right)^{k} C_{0}
$$

and then

$$
\sum_{k=1}^{\infty} \operatorname{prob}\left\{\omega \mid \sup _{t \in[0, T]}\left[\frac{\eta^{k}(\omega)}{k !} f_{k}(t, \omega)\right]>\frac{1}{k^{2}}\right\} \leq \sum_{k=1}^{\infty} k^{4}\left(C_{\eta}^{2} A\right)^{k} C_{0}
$$

By means of the D'Alembert Theorem, the fact that $0<C_{\eta}^{2} A<C_{\eta} A<1$ implies the convergence of the series $\sum_{k=1}^{\infty} k^{4}\left(C_{\eta}^{2} A\right)^{k} C_{0}$. The convergence of the series $\sum_{k=0}^{\infty} \frac{\eta^{k}(\omega)}{k !} f_{k}(t, \omega)$ (w.p.1) follows immediately by using the Borel-Cantelli lemma and the Weierstrass theorem. If the limit is denoted by $f(t, \omega)$, then its continuous property can be obtained easily from the assumption $f_{k}(t, \omega) \in C[0, T]$.

For the second moment, we have

$$
\begin{aligned}
\left\langle f^{2}(t, \omega)\right\rangle=\left\langle\left[\sum_{k=0}^{\infty} \frac{\eta^{k}(\omega)}{k !} f_{k}(t, \omega)\right]^{2}\right\rangle & \\
& \leq\left\langle\left[\sum_{k=0}^{\infty} \frac{C_{\eta}^{k}}{k !}\left|f_{k}(t, \omega)\right|\right]^{2}\right\rangle=\left\langle\left[\sum_{k=0}^{\infty} C_{\eta}^{k / 2} \frac{C_{\eta}^{k / 2}}{k !}\left|f_{k}(t, \omega)\right|\right]^{2}\right\rangle,
\end{aligned}
$$

and hence the boundedness

$$
\begin{aligned}
\left\langle f^{2}(t, \omega)\right\rangle \leq\left\langle\sum_{k=0}^{\infty} C_{\eta}^{k} \sum_{k=0}^{\infty} \frac{C_{\eta}^{k}}{k !^{2}} f_{k}^{2}(t, \omega)\right\rangle & \\
& \leq \frac{1}{1-C_{\eta}} \sum_{k=0}^{\infty}\left(C_{\eta} A\right)^{k} C_{0}=\frac{1}{1-C_{\eta}} \frac{C_{0}}{1-C_{\eta} A},
\end{aligned}
$$

where the Cauchy-Schwarz inequality has been used to derive the result.

The convergence of the series $\sum_{k=0}^{\infty} \frac{\eta^{k}(\omega)}{k !} \int_{0}^{T} f_{k}(t, \omega) d W(t, \omega)$ can be proved in a similar way, if the following inequality is noticed:

$$
\left\langle\left[\frac{\eta^{k}(\omega)}{k !} \int_{0}^{T} f_{k}(t, \omega) d W(t, \omega)\right]^{2}\right\rangle \leq \frac{C_{\eta}^{2 k}}{k !^{2}} \int_{0}^{T}\left\langle f_{k}^{2}(t, \omega)\right\rangle d t \leq\left(C_{\eta}^{2} A\right)^{k} C_{0} T
$$


Now we consider the last conclusion. Since

$$
\begin{aligned}
& \left\langle\left[\sum_{i=0}^{m-1} f\left(t_{i}, \omega\right)\left[W\left(t_{i+1}, \omega\right)-W\left(t_{i}, \omega\right)\right]-\sum_{k=0}^{\infty} \frac{\eta^{k}(\omega)}{k !} \int_{0}^{T} f_{k}(t, \omega) d W(t, \omega)\right]^{2}\right\rangle \\
& \quad=\left\langle\left[\sum_{k=0}^{\infty} \frac{\eta^{k}(\omega)}{k !}\left[\sum_{i=0}^{m-1} f_{k}\left(t_{i}, \omega\right)\left[W\left(t_{i+1}, \omega\right)-W\left(t_{i}, \omega\right)\right]-\int_{0}^{T} f_{k}(t, \omega) d W(t, \omega)\right]\right]^{2}\right\rangle \\
& \quad \leq \sum_{k=0}^{\infty} C_{\eta}^{k} \sum_{k=0}^{\infty} \frac{C_{\eta}^{k}}{k !^{2}}\left\langle\left[\sum_{i=0}^{m-1} f_{k}\left(t_{i}, \omega\right)\left[W\left(t_{i+1}, \omega\right)-W\left(t_{i}, \omega\right)\right]-\int_{0}^{T} f_{k}(t, \omega) d W(t, \omega)\right]^{2}\right\rangle \\
& \quad=\frac{1}{1-C_{\eta}} \sum_{k=0}^{\infty} \frac{C_{\eta}^{k}}{k !^{2}}\left\langle\left[\sum_{i=0}^{m-1} \int_{t_{i}}^{t_{i+1}}\left[f_{k}\left(t_{i}, \omega\right)-f_{k}(t, \omega)\right] d W(t, \omega)\right]^{2}\right\rangle \\
& \quad=\frac{1}{1-C_{\eta}} \sum_{k=0}^{\infty} \frac{C_{\eta}^{k}}{k !^{2}} \sum_{k=0}^{m-1} \int_{t_{i}}^{t_{i+1}}\left\langle\left[f_{k}\left(t_{i}, \omega\right)-f_{k}(t, \omega)\right]^{2}\right\rangle d t \\
& \quad \leq \frac{1}{1-C_{\eta}} \sum_{k=0}^{\infty} \frac{C_{\eta}^{k}}{k !^{2}} \sum_{i=0}^{m-1} A^{k}(k !)^{2} B\left(t_{i+1}-t_{i}\right)^{2} \\
& \quad \leq \frac{1}{1-C_{\eta}} \sum_{k=0}^{\infty}\left(C_{\eta} A\right)^{K} B T \Delta \\
& \quad=\Delta\left[\frac{B T}{1-C_{\eta}} \cdot \frac{1}{1-C_{\eta} A}\right],
\end{aligned}
$$

and as $\Delta=\max \left(t_{i+1}-t_{i}\right) \rightarrow 0$, the right side of the last equality tends to zero, we obtain the last conclusion immediately.

Now we are in a position to consider (1.2). Similar to other papers [2,3], for fixed $n$ we let $\lambda=\lambda_{n}+\eta$. Then (1.2) becomes

$$
\begin{aligned}
d u(t) & =\frac{v(t)}{p(t)} d t, \\
d v(t) & =\left[q(t)-\lambda_{n}-\eta\right] u(t) d t+\varepsilon u(t) d W(t, \omega), \\
u(0) & =0, \\
u(1) & =0 .
\end{aligned}
$$

To consider this boundary value problem by the shooting method, we first establish an initial value problem:

$$
\begin{aligned}
d u(t) & =\frac{v(t)}{p(t)} d t, \\
d v(t) & =\left[q(t)-\lambda_{n}-\eta\right] u(t) d t+\varepsilon u(t) d W(t, \omega), \\
u(0) & =0, \\
v(0) & =v_{0},
\end{aligned}
$$

where $v_{0}=p(0) u_{n}^{\prime}(0) \neq 0$ is the initial value of $v(t)$ corresponding to the deterministic eigenvalue problem for $\varepsilon=0$. In the remainder of this paper, we will omit the symbols $\omega$ and $t$ sometimes for simplicity. 
THEOREM 1. Suppose that

(1) for $t \in[0,1], p>0, p \in c^{\prime}, q \in c$;

(2) the Wiener process $W(t)$ satisfies the assumptions in Definition 1;

(3) there exist two positive constants $C_{\eta}$ and $A$ (to be specified later), such that for a given $F_{1}$-measurable bounded random variable $\eta$, we have

$$
|\eta| \leq C_{\eta}<\min \left(\frac{1}{A}, 1\right) \text { w.p.1. }
$$

Then for fixed $n$, the random initial value problem (2.3) has solutions $u$ and $v$. They satisfy all the assumptions of Remark 3, and have the series expansions:

$$
u(t)=\sum_{k=0}^{\infty} \frac{\eta^{k}}{k !} f_{k}(t), \quad v(t)=\sum_{k=0}^{\infty} \frac{\eta^{k}}{k !} g_{k}(t),
$$

where $f_{k}(t), g_{k}(t) \in H_{2}[0,1]$ are $F_{t}$-measurable, and the series expansions are convergent uniformly w.p.1.

Proof. To find the formal solution, we differentiate (2.3) $k$ times with respect to $\eta$, and then let $\eta=0$, so the equation (2.3) becomes

$$
\begin{aligned}
d u^{(k)}(t) & =\frac{v^{(k)}(t)}{p(t)} d t \\
d v^{(k)}(t) & =\left[q(t)-\lambda_{k}\right] u^{(k)}(t) d t-k u^{(k-1)}(t) d t+\varepsilon u^{(k)}(t) d W(t), \\
u^{(k)}(0) & =0, \\
v^{(k)}(0) & =0, \quad k=1,2, \ldots
\end{aligned}
$$

By Ito's theory we can show that there exist solutions $u^{(k)}(t)$ and $v^{(k)}(t)$ uniquely for (2.4), which are $F_{t}$-measurable and have continuous sample functions w.p.1. Furthermore we can have the following moments estimations:

$$
\begin{array}{cc}
\sup _{t \in[0,1]}\left\langle\left[u^{(k)}(t)\right]^{2}\right\rangle \leq A^{k} k !^{2} b_{0}, & \sup _{t \in[0,1]}\left\langle\left[v^{(k)}(t)\right]^{2}\right\rangle \leq A^{k} k !^{2} b_{0}^{*}, \\
\left\langle\sup _{t \in[0,1]}\left[u^{(k)}(t)\right]^{2}\right\rangle \leq A^{k} k !^{2} C_{0}, & \left\langle\sup _{t \in[0,1]}\left[v^{(k)}(t)\right]^{2}\right\rangle \leq A^{k} k !^{2} C_{0}^{*}, \\
\sup _{t \in[0,1]}\left\langle\left[u^{(k)}(t)\right]^{4}\right\rangle \leq A^{k} k !^{4} b_{0}, & \left\langle\sup _{t \in[0,1]}\left[u^{(k)}(t)\right]^{4}\right\rangle \leq A^{k} k !^{4} C_{0}, \\
\left\langle\left[u^{(k)}\left(t^{\prime}\right)-u^{(k)}\left(t^{\prime \prime}\right)\right]^{2}\right\rangle \leq A^{k} k !^{2} B\left|t^{\prime}-t^{\prime \prime}\right|,
\end{array}
$$

where $k=0,1, \ldots$, and $A, B, b_{0}, c_{0}, b_{0}^{*}, c_{0}^{*}$ are positive constants depending only on $p, q$, and $\varepsilon_{0}$.

In fact when $k=0, u^{(0)}$ and $v^{(0)}$ satisfy (2.3) for $\eta=0$. Its integral form is

$$
\left\{\begin{array}{l}
u^{(0)}(t)=\int_{0}^{t} \frac{v^{(0)}}{p} d t \\
v^{(0)}(t)=v_{0}+\int_{0}^{t}\left(q-\lambda_{n}\right) u^{(0)} d t+\varepsilon \int_{0}^{t} u^{(0)} d W(t) .
\end{array}\right.
$$

Then

$$
u^{(0)}(t)=v_{0} \int_{0}^{t} \frac{1}{p} d t+\int_{0}^{t} d \tau\left(\frac{1}{p} \int_{0}^{\tau}\left(q-\lambda_{n}\right) u^{(0)} d s\right)+\varepsilon \int_{0}^{t} d \tau\left(\frac{1}{p} \int_{0}^{\tau} u^{(0)} d W(s)\right)
$$


Using the notations

$$
Q_{M}=\operatorname{Max}_{t \in[0,1]}\left|q(t)-\lambda_{n}\right|, \quad p_{m}=\underset{t \in[0,1]}{\operatorname{Min}} p(t),
$$

we can have

$$
\begin{aligned}
{\left[u^{(0)}(t)\right]^{2} } & \leq 3\left\{\frac{v_{0}^{2}}{p_{m}^{2}}+\frac{Q_{M}^{2}}{p_{m}^{2}}\left[\int_{0}^{t} d \tau \int_{0}^{\tau}\left|u^{(0)}\right| d s\right]^{2}+\varepsilon^{2} \frac{1}{p_{m}^{2}}\left[\int_{0}^{t} d \tau\left|\int_{0}^{\tau} u^{(0)} d W(s)\right|\right]^{2}\right\} \\
& \leq 3\left\{\left(\frac{v_{0}}{p_{m}}\right)^{2}+\left(\frac{Q_{M}}{p_{m}}\right)^{2} \int_{0}^{t}\left[u^{(0)}\right]^{2} d s+\left(\frac{\varepsilon}{p_{m}}\right)^{2} \int_{0}^{t} d \tau\left[\int_{0}^{\tau} u^{(0)} d W(s)\right]^{2}\right\} .
\end{aligned}
$$

It follows that

$$
\left\langle\left[u^{(0)}(t)\right]^{2}\right\rangle \leq 3\left\{\left(\frac{v_{0}}{p_{m}}\right)^{2}+\frac{Q_{M}^{2}+\varepsilon_{0}^{2}}{p_{m}^{2}} \int_{0}^{t}\left\langle\left[u^{(0)}\right]^{2}\right\rangle d S\right\},
$$

and by Gronwall's inequality we find that

$$
\sup _{t \in[0,1]}\left\langle\left[u^{(0)}\right]^{2}\right\rangle \leq 3\left(\frac{v_{0}}{p_{m}}\right)^{2} \exp \left[3 \frac{Q_{M}^{2}+\varepsilon_{0}^{2}}{p_{m}^{2}}\right] \doteq b_{0}
$$

From (2.6) it also follows that

$$
\begin{aligned}
& \left\langle\sup _{t \in[0,1]}\left[u^{(0)}(t)\right]^{2}\right\rangle \\
& \quad \leq 3\left\langle\left(\frac{v_{0}}{p_{m}}\right)^{2}+\left(\frac{Q_{M}}{p_{m}}\right)^{2} \int_{0}^{1}\left[u^{(0)}\right]^{2} d s+\left(\frac{\varepsilon}{p_{m}}\right)^{2} \int_{0}^{1} d \tau\left[\int_{0}^{\tau} u^{(0)} d W(s)\right]^{2}\right\rangle \\
& \quad \leq 3\left\{\left(\frac{v_{0}}{p_{m}}\right)^{2}+\left(\frac{Q_{M}}{p_{m}}\right)^{2} \int_{0}^{1}\left\langle\left[u^{(0)}\right]^{2}\right\rangle d s+\left(\frac{\varepsilon_{0}}{p_{m}}\right)^{2} \int_{0}^{1}\left\langle\left[u^{(0)}\right]^{2}\right\rangle d s\right\},
\end{aligned}
$$

and then from (2.7) we have

$$
\left\langle\sup _{t \in[0,1]}\left[u^{(0)}(t)\right]^{2}\right\rangle \leq 3\left\{\left(\frac{v_{0}}{p_{m}}\right)^{2}+b_{0} \frac{Q_{M}^{2}+\varepsilon_{0}^{2}}{p_{m}^{2}}\right\} \doteq C_{0} .
$$

When $k=1,2, \ldots$, the equation (2.4) is linear. So by a way very similar to one given by Arnold [12], we can have

$$
\left(\begin{array}{l}
u^{(k)} \\
v^{(k)}
\end{array}\right)=\Phi^{e}(t) \int_{0}^{t} \Phi^{e^{-1}}(\tau)\left(\begin{array}{c}
0 \\
-k u^{(k-1)}
\end{array}\right) d \tau
$$

where $\Phi^{e}$ is the fundamental solution matrix satisfying the equation

$$
\left\{\begin{array}{l}
d \Phi^{\varepsilon}(t)=\left(\begin{array}{cc}
0 & \frac{1}{p} \\
q-\lambda_{n} & 0
\end{array}\right) \Phi^{\varepsilon}(t) d t+\left(\begin{array}{ll}
0 & 0 \\
\varepsilon & 0
\end{array}\right) \Phi^{\varepsilon}(t) d W(t), \\
\Phi^{\varepsilon}(0)=\left(\begin{array}{ll}
1 & 0 \\
0 & 1
\end{array}\right) .
\end{array}\right.
$$

By Ito's theory we know that the $F_{t}$-measurable solution $\Phi^{\varepsilon}(t)$ of $(2.9)$ exists uniquely. It is straightforward to show that $d\left[\operatorname{Det} \Phi^{e}(t)\right]=0$. Then the fact that $\operatorname{Det} \Phi^{\varepsilon}(t)=$ Det $\Phi^{e}(0)=1 \neq 0$, w.p. 1 yields the existence of $\Phi^{e^{-1}}(t)$ in $(2.8)$. The $F_{t}$-measurability 
of $u^{(k)}, v^{(k)}$ in (2.8) can be obtained by induction if the $F_{t}$-measurability of $\Phi^{\varepsilon^{-1}}(t)$ is noticed.

To prove (2.5), we rewrite (2.4) in the integral form

$$
\begin{aligned}
u^{(k)}(t)=\int_{0}^{t} \frac{v^{(k)}}{p} d t= & \int_{0}^{t} d \tau\left[\frac{1}{p} \int_{0}^{\tau}\left(q-\lambda_{n}\right) u^{(k)} d s\right] \\
& +\varepsilon \int_{0}^{t} d \tau\left[\frac{1}{p} \int_{0}^{\tau} u^{(k)} d W(s)\right]-k \int_{0}^{t} d \tau\left[\frac{1}{p} \int_{0}^{\tau} u^{(k-1)} d s\right] .
\end{aligned}
$$

Similar to (2.6), we can have

$$
\begin{aligned}
{\left[u^{(k)}\right]^{2} \leq 3\left\{\left(\frac{Q_{M}}{p_{m}}\right)^{2} \int_{0}^{t}\left[u^{(k)}\right]^{2} d s+\left(\frac{\varepsilon}{p_{m}}\right)^{2}\right.} & \int_{0}^{t} d \tau\left[\int_{0}^{\tau} u^{(k)} d W(s)\right]^{2} \\
& \left.+\left(\frac{k}{p_{m}}\right)^{2} \int_{0}^{t}\left[u^{(k-1)}\right]^{2} d s\right\},
\end{aligned}
$$

and

$$
\left\langle\left[u^{(k)}\right]^{2}\right\rangle \leq 3\left\{\frac{Q_{M}^{2}+\varepsilon_{0}^{2}}{p_{m}^{2}} \int_{0}^{t}\left\langle\left[u^{(k)}\right]^{2}\right\rangle d s+\left(\frac{k}{p_{m}}\right)^{2} \int_{0}^{t}\left\langle\left[u^{(k-1)}\right]^{2}\right\rangle d s\right\} .
$$

Then the first estimation in $(2.5 \mathrm{a})$ can be proved by using Gronwall's inequality and the induction method, where $A=\left(3 / p_{m}^{2}\right) \exp \left[3\left(Q_{M}^{2}+\varepsilon_{0}^{2}\right) / p_{m}^{2}\right]$. The other estimations in (2.5) can also be proved in a similar way.

Finally, by using (2.5), Remark 3 , and taking the limit $m \rightarrow \infty$ for equations satisfied by $U^{(m)}=\sum_{k=0}^{m} \frac{\eta^{k} !}{k !} u^{(k)}, V^{(m)}=\sum_{k=0}^{m} \frac{\eta_{k}^{k}}{k !} v^{(k)}$ :

$$
\left\{\begin{array}{l}
U^{(m)}(t)=\int_{0}^{t} \frac{1}{p} V^{(m)} d t, \\
V^{(m)}(t)=v_{0}+\int_{0}^{t}\left(q-\lambda_{n}\right) U^{(m)} d t-\eta \int_{0}^{t} U^{(m-1)} d t+\varepsilon \int_{0}^{t} U^{(m)} d W(t),
\end{array}\right.
$$

we can easily prove the conclusion that the formal solutions

$$
u(t)=\sum_{k=0}^{\infty} \frac{\eta^{k}}{k !} u^{(k)}(t), \quad v(t)=\sum_{k=0}^{\infty} \frac{\eta^{k}}{k !} v^{(k)}(t),
$$

exist with continuous sample functions w.p.1, and that they satisfy equation (2.3). Therefore it completes our proof.

Remark 4.

(1) There exists a constant $C_{1}$, independent of $\varepsilon$, such that for the solution $u$ of (2.3), we have

$$
\left\langle\left[\int_{0}^{t} u d W(t)\right]^{2}\right\rangle \leq C_{1}
$$

(2) If $\eta_{1}, \eta_{2}$ are two random variables satisfying assumption (3) of Theorem 1, $\Omega_{\eta}=\left\{\omega \mid \eta_{2} \neq \eta_{1}\right\}$, and $\chi\left(\Omega_{\eta}\right)$ is the indicator of $\Omega_{\eta}$, then there exists a constant $C_{2}$, independent of $\varepsilon$, such that

$$
\left\langle\left[\frac{1}{\eta_{2}-\eta_{1}} \chi\left(\Omega_{\eta}\right) \int_{0}^{t}\left[u\left(t, \eta_{2}\right)-u\left(t, \eta_{1}\right)\right] d W(t)\right]^{2}\right\rangle \leq C_{2},
$$


where the notation $u(t, \eta)$ is introduced for the solution $u$ of $(2.3)$ to emphasize the dependence of $\eta$.

Proof. To prove (2.12), we expand $u\left(t, \eta_{2}\right), u\left(t, \eta_{1}\right)$ in series, thus

$$
\begin{array}{r}
\frac{1}{\eta_{2}-\eta_{1}} \chi\left(\Omega_{\eta}\right) \int_{0}^{t}\left[u\left(t, \eta_{2}\right)-u\left(t, \eta_{1}\right)\right] d W(t) \\
=\sum_{k=0}^{\infty} \frac{\eta_{2}^{k}-\eta_{1}^{k}}{\eta_{2}-\eta_{1}} \frac{1}{k !} \chi\left(\Omega_{\eta}\right) \int_{0}^{t} u^{(k)} d W(t) \\
=\chi\left(\Omega_{\eta}\right) \sum_{k=1}^{\infty} \frac{\eta_{2}^{k}-\eta_{1}^{k}}{\eta_{2}-\eta_{1}} \frac{1}{k !} \int_{0}^{t} u^{(k)} d W(t),
\end{array}
$$

so that

$$
\begin{gathered}
\left\langle\left[\frac{1}{\eta_{2}-\eta_{1}} \chi\left(\Omega_{\eta}\right) \int_{0}^{t}\left[u\left(t, \eta_{2}\right)-u\left(t, \eta_{1}\right)\right] d W(t)\right]^{2}\right\rangle \\
\leq\left\langle\left[\sum_{k=1}^{\infty} k\left(C_{\eta}\right)^{k-1} \frac{1}{k !}\left|\int_{0}^{t} u^{(k)} d W(t)\right|\right]^{2}\right\rangle \\
\quad \leq \sum_{k=0}^{\infty} C_{\eta}^{k} \sum_{k=0}^{\infty} \frac{C_{\eta}^{k}}{k !^{2}} \int_{0}^{t}\left\langle\left[u^{(k+1)}\right]^{2}\right\rangle d t \\
\leq \frac{1}{1-C_{\eta}} \sum_{k=0}^{\infty} C_{\eta}^{k} A^{k+1}(k+1)^{2} C_{0} .
\end{gathered}
$$

Since we assume $C_{\eta} A<1$, then D'Alembert's theorem yields the convergence of the last series. (2.12) follows directly. The proof for (2.11) can be obtained in a similar way.

3. Random mapping and solutions of the eigenvalue problem. Let $\Phi(t)$ be the deterministic solution of $(2.9)$ for $\varepsilon=0$. It satisfies the equation

$$
\frac{d \Phi}{d t}=\left(\begin{array}{cc}
0 & 1 / p \\
q-\lambda_{n} & 0
\end{array}\right) \Phi, \quad \Phi(0)=\left(\begin{array}{ll}
1 & 0 \\
0 & 1
\end{array}\right),
$$

and has the form

$$
\Phi(t)=\left(\begin{array}{ll}
\varphi_{11}(t) & \varphi_{12}(t) \\
\varphi_{21}(t) & \varphi_{22}(t)
\end{array}\right)=\left(\begin{array}{cc}
\varphi_{11}(t) & u_{n}(t) / v_{0} \\
\varphi_{21}(t) & p(t) u_{n}^{\prime}(t) / v_{0}
\end{array}\right) .
$$

By Liouville's theorem we know that $\operatorname{Det} \Phi(t)=\operatorname{Det} \Phi(0)=1$ or $\operatorname{Det} \Phi(1)=1$. Hence the boundary condition $u_{n}(1)=0$ implies that $\varphi_{11}(1)=v_{0} /\left[p(1) u_{n}^{\prime}(1)\right] \neq 0$. Now we define a random mapping similar to one in $[8,13]$ under the conditions of Theorem 1:

$$
T(\eta)=\eta-\left[v_{0} \varphi_{11}^{-1}(1)\right] u(1, \eta),
$$

where $u(t, \eta)$ is the solution of (2.3). If we can prove that $T(\eta)$ is a contraction mapping defined on a suitable metric space, then by the well-known theorem there exists a unique random fixed point $\hat{\eta}$ such that $T(\hat{\eta})=\hat{\eta}$, and thereby $u(1, \hat{\eta})=0$, or $u(t, \hat{\eta})$ is the solution of the random eigenvalue problem (2.2). Unfortunately this argument is not always valid for all $\omega \in \Omega$ in the general case, but when $\varepsilon$ is small we 
can show that it is true for all $\omega \in \mathbb{C}_{C_{\eta}}$, where $\mathcal{C}_{\eta}$ is an event with large probability and will be defined below. For fixed $\varepsilon, C_{\eta}>0$ we define 3 events:

(1) $\mathscr{C}_{\eta}=\left\{\omega \mid T\right.$ is a contraction mapping on the ball of radius $C_{\eta}$ about zero, with contraction $\left.\frac{1}{2}\right\}$, that is, for all $F_{1}$-measurable random variables $\eta_{1}, \eta_{2}$ such that $\left|\eta_{i}\right| \leq C_{\eta}, \mathscr{C}_{C_{\eta}}$ is the event that $\left|T\left(\eta_{i}\right)\right| \leq C_{\eta}$, and $\left|T\left(\eta_{2}\right)-T\left(\eta_{1}\right)\right| \leq \frac{1}{2}\left|\eta_{2}-\eta_{1}\right|$;

(2) $\mathscr{B}_{C_{\eta}}^{\varepsilon}=\left\{\omega \mid \exists \eta^{*}: \eta^{*}\right.$ is $F_{1}$-measurable, $\left.\left|\eta^{*}\right| \leq C_{\eta},\left|T\left(\eta^{*}\right)\right|>C_{\eta}\right\}$;

(3) $\mathscr{C}_{C_{\eta}}^{\varepsilon}=\left\{\omega \mid \exists \eta_{1}^{*}, \eta_{2}^{*}: \eta_{i}^{*}\right.$ is $F_{1}$-measurable, $\left.\left|\eta_{i}^{*}\right| \leq C_{\eta},\left|T\left(\eta_{2}^{*}\right)-T\left(\eta_{1}^{*}\right)\right|>\frac{1}{2}\left|\eta_{2}^{*}-\eta_{1}^{*}\right|\right\}$. Then for $C_{\eta}$ we can have

THEOREM 2. Under the assumptions 1, 2 of Theorem 1, there exist three positive constants $A, C_{T}, C_{8}$, depending only on $p, q, \varepsilon_{0}$, and two constants $C_{\eta}, C_{A}$, independent of $\varepsilon$, such that

$$
0<C_{\eta}<\min \left(1, \frac{1}{A}, \frac{1}{2 C_{T}}, \frac{1}{2 C_{8}}\right),
$$

and

$$
\operatorname{Prob}\left\{C_{\eta}\right\} \geq 1-C_{A} \varepsilon^{2} \text {. }
$$

Proof. Under the assumptions 1, 2 of Theorem 1, if we select $A$ to be a constant in (2.5), and $C_{T}, C_{8}$ to be suitable constants which will be specified later in the proof of this theorem, then for the constant $C_{\eta}$ and $F_{1}$-measurable random variable $\eta$ satisfying the conditions

$$
0<C_{\eta}<\min \left(1, \frac{1}{A}, \frac{1}{2 C_{T}}, \frac{1}{2 C_{8}}\right) \text { and }|\eta| \leq C_{\eta} \quad \text { w.p.1, }
$$

we can discuss equation (2.3) by Theorem 1 and Remark 4. To prove this theorem we need some properties for the solutions of $(2.3)$ for $\varepsilon=0$. If we denote them by $\bar{u}, \bar{v}$, then we have the equations

$$
\left\{\begin{array}{l}
d \bar{u}=(\bar{v} / p) d t \\
d \bar{v}=\left(q-\lambda_{n}-\eta\right) \bar{u} d t \\
\bar{u}(0)=0 \\
\bar{v}(0)=v_{0}
\end{array}\right.
$$

By setting $\bar{k}=\max _{t \in[0,1]}|q(t)|+\left|\lambda_{k}\right|+1$, we obtain

$$
|\bar{u}(t)| \leq \int_{0}^{t} \frac{|\bar{v}|}{p} d t, \quad|\bar{v}(t)| \leq\left|v_{0}\right|+\int_{0}^{t} \bar{k}|\bar{u}| d t,
$$

or

$$
|\bar{u}(t)| \leq \frac{\left|v_{0}\right|}{p_{m}}+\frac{\bar{k}}{p_{m}} \int_{0}^{t} d \tau \int_{0}^{\tau}|\bar{u}| d s \leq \frac{\left|v_{0}\right|}{p_{m}}+\frac{\bar{k}}{p_{m}} \int_{0}^{t}|\bar{u}| d s ;
$$

hence Gronwall's inequality yields a constant $C_{3}$ depending only on $p, q$, such that

$$
\sup _{t \in[0,1]}|\bar{u}(t)| \leq \frac{\left|v_{0}\right|}{p_{m}} e^{k / p_{m}} \doteq C_{3}
$$


Subtracting (3.5) from (2.3), we obtain the equations for $u-\bar{u}, v-\bar{v}$ :

$$
\begin{aligned}
d(u-\bar{u}) & =\frac{v-\bar{v}}{p} d t, \\
d(v-\bar{v}) & =\left(q-\lambda_{n}-\eta\right)(u-\bar{u}) d t+\varepsilon u d W(t), \\
u(0)-\bar{u}(0) & =0, \\
v(0)-\bar{v}(0) & =0 .
\end{aligned}
$$

If we rewrite this equation in integral form, and let

$$
\alpha(t)=\frac{1}{p_{m}} \int_{0}^{t} d \tau\left|\int_{0}^{\tau} u d W(s)\right|,
$$

then Gronwall's inequality and (2.11) give the estimations

$$
|u(t)-\bar{u}(t)| \leq \varepsilon\left[\alpha(t)+\frac{\bar{k}}{p_{m}} \int_{0}^{t} \alpha(s) e^{k(t-s) / p_{m}} d s\right],
$$

and

$$
\sup _{t \in[0,1]}\left\langle(u-\bar{u})^{2}\right\rangle \leq C_{4} \varepsilon^{2}
$$

where $C_{4}$ is a constant independent of $\varepsilon$.

By a similar argument we can find a constant $C_{5}$ depending only on $p, q$ and a constant $C_{6}$, independent of $\varepsilon$, such that

$$
\begin{gathered}
\sup _{t \in[0,1]}\left|\bar{u}\left(t, \eta_{2}\right)-\bar{u}\left(t, \eta_{1}\right)\right| \leq\left|\eta_{2}-\eta_{1}\right| C_{3} \frac{e^{k / p_{m}}}{p_{m}}=\left|\eta_{2}-\eta_{1}\right| C_{5}, \\
\sup _{t \in[0,1]}\left\langle\left\{\chi\left(\Omega_{\eta}\right) \frac{\left[u\left(t, \eta_{2}\right)-u\left(t, \eta_{1}\right)\right]-\left[\bar{u}\left(t, \eta_{2}\right)-\bar{u}\left(t, \eta_{1}\right)\right]}{\eta_{2}-\eta_{1}}\right\}^{2}\right\rangle \leq C_{6} \varepsilon^{2} .
\end{gathered}
$$

Now we consider the probability of $\mathscr{A}_{C_{\eta}}$. Since $\left(\mathscr{A}_{C_{\eta}}\right)^{C} \subset \mathscr{B}_{C_{\eta}}^{\varepsilon} \cup \mathscr{C}_{C_{\eta}}^{\varepsilon}$, the proof of this theorem can be obtained by finding constants $C_{\eta}, C_{B}, C_{C}$, independent of $\varepsilon$, such that

$$
\operatorname{prob}\left\{\mathscr{B}_{C_{\eta}}^{\varepsilon}\right\} \leq C_{B} \varepsilon^{2}, \quad \operatorname{prob}\left\{\mathscr{C}_{C_{\eta}}^{\varepsilon}\right\} \leq C_{C} \varepsilon^{2} .
$$

To prove (3.11) we first rewrite $T(\eta)$ in another form. If we notice that $\bar{u}-u_{n}$ and $v-v_{n}$ satisfy the linear equations:

$$
\begin{aligned}
d\left(\bar{u}-u_{n}\right) & =\frac{\bar{v}-v_{n}}{p} d t, \\
d\left(\bar{v}-v_{n}\right) & =\left(q-\lambda_{n}\right)\left(\bar{u}-u_{n}\right) d t-\eta \bar{u} d t, \\
\bar{u}(0)-u_{n}(0) & =0 \\
v(0)-v_{n}(0) & =0
\end{aligned}
$$

then we easily have

$$
\left(\begin{array}{c}
\bar{u}(t)-u_{n}(t) \\
\bar{v}(t)-v_{n}(t)
\end{array}\right)=\Phi(t) \int_{0}^{t} \Phi^{-1}(t)\left(\begin{array}{c}
0 \\
-\eta \bar{u}
\end{array}\right) d t,
$$

where $\Phi(t)$ is the fundamental solution matrix (3.2). It follows that

$$
\bar{u}(t)-u_{n}(t)=\frac{\eta}{v_{0}}\left[\varphi_{11}(t) \int_{0}^{t} u_{n} \bar{u} d t-u_{n}(t) \int_{0}^{t} \varphi_{11} \bar{u} d t\right]
$$


and then

$$
\begin{aligned}
\bar{u}(1, \eta) & =u_{n}(1)+\frac{\eta}{v_{0}}\left[\varphi_{11}(1) \int_{0}^{1} u_{n} \bar{u} d t-u_{n}(1) \int_{0}^{1} \varphi_{11} \bar{u} d t\right] \\
& =\frac{\eta}{v_{0}} \varphi_{11}(1) \int_{0}^{1} u_{n} \bar{u} d t .
\end{aligned}
$$

Hence the random mapping becomes

$$
\begin{aligned}
T(\eta) & =\eta-\left[v_{0} \varphi_{11}^{-1}(1)\right] u(1, \eta) \\
& =\varphi_{11}^{-1}(1)[\bar{u}(1, \eta)-u(1, \eta)] v_{0}+\varphi_{11}^{-1}(1)\left[\varphi_{11}(1) \eta-\bar{u}(1, \eta) v_{0}\right] \\
& =\varphi_{11}^{-1}(1)[\bar{u}(1, \eta)-u(1, \eta)] v_{0}+\eta\left[1-\int_{0}^{1} u_{n} \bar{u} d t\right] \\
& =\varphi_{11}^{-1}(1)[\bar{u}(1, \eta)-u(1, \eta)] v_{0}+\eta \int_{0}^{1} u_{n}\left(u_{n}-\bar{u}\right) d t,
\end{aligned}
$$

where the normalized condition (1.3) has been used to derive the last equality.

Using (3.7), (3.13), we have

$$
\begin{aligned}
|T(\eta)| \leq \varepsilon\left|\varphi_{11}^{-1}(1)\right|\left|v_{0}\right|[\alpha(1) & \left.+\frac{\bar{k}}{p_{m}} \int_{0}^{1} \alpha(s) e^{\bar{k}(1-s) / p_{m}} d s\right] \\
& +\frac{\eta^{2}}{\left|v_{0}\right|}\left|\int_{0}^{1}\left[u_{n} \varphi_{11}(t) \int_{0}^{t} u_{n} \bar{u} d s-u_{n}^{2} \int_{0}^{t} \varphi_{11} \bar{u} d s\right] d t\right|,
\end{aligned}
$$

and then

$$
|T(\eta)| \leq \varepsilon \beta+C_{T}^{*} \eta^{2} \leq \varepsilon \beta+C_{T} \eta^{2},
$$

where (3.6) has been used, and

$$
\begin{aligned}
\beta & =\left|\varphi_{11}^{-1}(1)\right|\left|v_{0}\right|\left[\alpha(1)+\frac{\bar{k}}{p_{m}} \int_{0}^{1} \alpha(s) e^{\bar{k}(1-s) / p_{m}} d s\right], \\
C_{T}^{*} & =\frac{1}{\left|v_{0}\right|} \int_{0}^{1}\left[\left|u_{n}\right|\left|\varphi_{11}(t)\right| \int_{0}^{t} C_{3}\left|u_{n}\right| d s+u_{n}^{2} \int_{0}^{t} C_{3}\left|\varphi_{11}\right| d s\right] d t, \\
C_{T} & =\max \left(1, C_{T}^{*}\right) .
\end{aligned}
$$

The constants $A$ and $C_{T}$ depend only on $p, q, \varepsilon_{0}$. So we can find constants $l_{1}, C_{\eta}$, independent of $\varepsilon$, such that

$$
0<l_{1} \leq C_{\eta}<\min \left(1, \frac{1}{A}, \frac{1}{2 C_{T}}\right),
$$

and

$$
|T(\eta)| \leq \varepsilon \beta+\frac{1}{2} C_{\eta} \quad\left(|\eta| \leq C_{\eta}\right) .
$$


The upper bound for the second moment of $\beta$ can be obtained by means of (2.11) and the expression of $\alpha$ :

$$
\begin{aligned}
\left\langle\beta^{2}\right\rangle & =\left[\varphi_{11}^{-1}(1) v_{0}\right]^{2}\left\langle\left[\alpha(1)+\frac{\bar{k}}{p_{m}} \int_{0}^{1} \alpha(s) e^{\bar{k}(1-s) / p_{m}} d s\right]^{2}\right\rangle \\
& \leq 2\left[\varphi_{11}^{-1}(1) v_{0}\right]^{2}\left\{\left\langle\alpha^{2}(1)\right\rangle+\left(\frac{\bar{k}}{p_{m}}\right)^{2} \int_{0}^{1}\left\langle\alpha^{2}(s)\right\rangle e^{2 \bar{k}(1-s) / p_{m}} d s\right\} \\
& \leq C_{\beta},
\end{aligned}
$$

where the constant $C_{\beta}$ is independent of $\varepsilon$. For $\omega \in \mathscr{B}_{C_{\eta}}^{\varepsilon}$, (3.16) implies $C_{\eta}<$ $\varepsilon \beta+C_{\eta} / 2$ or $C_{\eta} / 2<\varepsilon \beta$. Then

$$
\operatorname{prob}\left\{\mathscr{B}_{C_{\eta}}^{\varepsilon}\right\} \leq \frac{\left\langle(\varepsilon \beta)^{2}\right\rangle}{\left(C_{\eta} / 2\right)^{2}} \leq \frac{4 C_{\beta}}{l_{1}^{2}} \varepsilon^{2} \doteq C_{\beta} \varepsilon^{2} .
$$

This is the first estimation of (3.11). Now we consider the second one. First from (3.13) we can have the following equality:

$$
\bar{u}\left(1, \eta_{2}\right)-\bar{u}\left(1, \eta_{1}\right)=\frac{\varphi_{11}(1)}{v_{0}} \int_{0}^{1} u_{n}\left[\left(\eta_{2}-\eta_{1}\right) \bar{u}\left(t, \eta_{1}\right)+\eta_{2}\left(\bar{u}\left(t, \eta_{2}\right)-\bar{u}\left(t, \eta_{1}\right)\right)\right] d t .
$$

For $\omega \in \Omega_{\eta}$ or $\eta_{2} \neq \eta_{1}$, since

$$
\begin{aligned}
& \left|\varphi_{11}(1)-\frac{\bar{u}\left(1, \eta_{2}\right)-\bar{u}\left(1, \eta_{1}\right)}{\eta_{2}-\eta_{1}} v_{0}\right| \\
& \quad \leq\left|\varphi_{11}(1)\right|\left|1-\int_{0}^{1} u_{n}\left[\bar{u}\left(t, \eta_{1}\right)+\frac{\eta_{2}\left(\bar{u}\left(t, \eta_{2}\right)-\bar{u}\left(t, \eta_{1}\right)\right)}{\eta_{2}-\eta_{1}}\right] d t\right| \\
& \quad \leq\left|\varphi_{11}(1)\right| \int_{0}^{1}\left|u_{n}\right|\left[\left|u_{n}-\bar{u}\left(t, \eta_{1}\right)\right|+\left|\eta_{2}\right|\left|\frac{\bar{u}\left(t, \eta_{2}\right)-\bar{u}\left(t, \eta_{1}\right)}{\eta_{2}-\eta_{1}}\right|\right] d t,
\end{aligned}
$$

we can find constants $C_{7}, C_{8}$ depending only on $p, q, \varepsilon_{0}$, such that

$$
\begin{aligned}
& \left|\varphi_{11}(1)-\frac{\bar{u}\left(1, \eta_{2}\right)-\bar{u}\left(1, \eta_{1}\right)}{\eta_{2}-\eta_{1}} v_{0}\right| \\
& \leq\left|\varphi_{11}(1)\right| \int_{0}^{1}\left|u_{n}\right|\left[C_{5} C_{\eta}+C_{7} C_{\eta}\right] d t \doteq C_{8} C_{\eta}\left|\varphi_{11}(1)\right|,
\end{aligned}
$$

where (3.9), (3.13), and (3.6) are used to derive the first inequality.

For $\eta_{2}^{*}, \eta_{1}^{*}$ in the definition of the event $\mathscr{C}_{C_{\eta}}^{\varepsilon}$, let $\Omega_{\eta^{*}}=\left\{\omega \mid \eta_{2}^{*} \neq \eta_{1}^{*}\right\}$, and

$$
u^{*}=\frac{\left[u\left(1, \eta_{2}^{*}\right)-u\left(1, \eta_{1}^{*}\right)\right]-\left[\bar{u}\left(1, \eta_{2}^{*}\right)-\bar{u}\left(1, \eta_{1}^{*}\right)\right]}{\eta_{2}^{*}-\eta_{1}^{*}} \quad\left(\omega \in \Omega_{\eta^{*}}\right) .
$$

Then for $\omega \in \mathscr{C}_{C_{\eta}}^{\varepsilon}$, we know from (3.10) that

$$
\left\langle\left[\chi\left(\Omega_{\eta^{*}}\right) u^{*}\right]^{2}\right\rangle \leq C_{6} \varepsilon^{2},
$$


and that

$$
\begin{aligned}
\mid T\left(\eta_{2}^{*}\right) & -T\left(\eta_{1}^{*}\right) \mid \\
= & \left|\left[\eta_{2}^{*}-\varphi_{11}^{-1}(1) v_{0} u\left(1, \eta_{2}^{*}\right)\right]-\left[\eta_{1}^{*}-\varphi_{11}^{-1}(1) v_{0} u\left(1, \eta_{1}^{*}\right)\right]\right| \\
= & \left|\eta_{2}^{*}-\eta_{1}^{*}\right|\left|\varphi_{11}^{-1}(1)\right| \mid \varphi_{11}(1)-v_{0} \frac{\bar{u}\left(1, \eta_{2}^{*}\right)-\bar{u}\left(1, \eta_{1}^{*}\right)}{\eta_{2}^{*}-\eta_{1}^{*}} \\
& -v_{0} \frac{\left[u\left(1, \eta_{2}^{*}\right)-u\left(1, \eta_{1}^{*}\right)\right]-\left[\bar{u}\left(1, \eta_{2}^{*}\right)-\bar{u}\left(1, \eta_{1}^{*}\right)\right]}{\eta_{2}^{*}-\eta_{1}^{*}} \mid \\
\leq & \left|\eta_{2}^{*}-\eta_{1}^{*}\right|\left|\varphi_{11}^{-1}(1)\right|\left[\left|\varphi_{11}(1)-v_{0} \frac{\bar{u}\left(1, \eta_{2}^{*}\right)-\bar{u}\left(1, \eta_{1}^{*}\right)}{\eta_{2}^{*}-\eta_{1}^{*}}\right|+\left|v_{0}\right|\left|u^{*}\right|\right] \\
\leq & \left|\eta_{2}^{*}-\eta_{1}^{*}\right|\left|\varphi_{11}^{-1}(1)\right|\left[\left|\varphi_{11}(1)\right| C_{8} C_{\eta}+\left|v_{0}\right|\left|u^{*}\right|\right],
\end{aligned}
$$

where (3.19) has been used to obtain the last inequality. When $\omega \in \mathscr{C}_{C_{\eta}}$, we know that $\frac{1}{2}\left|\eta_{2}^{*}-\eta_{1}^{*}\right|<\left|T\left(\eta_{2}^{*}\right)-T\left(\eta_{1}^{*}\right)\right|$. Then the above inequality implies

$$
\frac{1}{2}\left|\eta_{2}^{*}-\eta_{1}^{*}\right|<\left|\eta_{2}^{*}-\eta_{1}^{*}\right|\left[C_{8} C_{\eta}+\left|\varphi_{11}^{-1}(1)\right|\left|v_{0}\right|\left|u^{*}\right|\right] \text {, }
$$

i.e.,

or

$$
\frac{1}{2}<C_{8} C_{\eta}+\left|\varphi_{11}^{-1}(1)\right|\left|v_{0}\right|\left|u^{*}\right|
$$

$$
\left(\frac{1}{2}-C_{8} C_{\eta}\right) \frac{1}{\left|v_{0}\right|}\left|\varphi_{11}(1)\right|<\left|u^{*}\right|
$$

If we select two constants $l_{2}, C_{\eta}$, independent of $\varepsilon$, such that

$$
\begin{aligned}
& 0<C_{\eta}<\min \left(1, \frac{1}{A}, \frac{1}{2 C_{T}}, \frac{1}{2 C_{8}}\right), \\
& 0<l_{2}<\left(\frac{1}{2}-C_{8} C_{\eta}\right) \frac{1}{\left|v_{0}\right|}\left|\varphi_{11}(1)\right|,
\end{aligned}
$$

then the fact that $\mathscr{C}_{C_{\eta}} \subset \Omega_{\eta^{*}}$ gives the estimation

$$
\begin{aligned}
\operatorname{prob}\left\{\mathscr{C}_{C_{\eta}}^{\epsilon}\right\} & \leq \operatorname{prob}\left\{\omega\left|0<l_{2}<\right| \chi\left(\Omega_{\eta^{*}}\right) u^{*} \mid\right\} \\
& \leq \frac{1}{l_{2}^{2}}\left\langle\left[\chi\left(\Omega_{\eta^{*}}\right) u^{*}\right]^{2}\right\rangle \leq \frac{C_{6}}{l_{2}^{2}} \varepsilon^{2} \doteq C_{c} \varepsilon^{2} .
\end{aligned}
$$

Combining it with (3.18), we obtain

$$
\operatorname{prob}\left\{C_{C_{\eta}}\right\} \geq 1-\left(C_{B}+C_{C}\right) \varepsilon^{2} \doteq 1-C_{A} \varepsilon^{2},
$$

and this completes our proof.

4. Approximation and expansion. In order to discuss the properties of the solutions, we need the following lemmas:

LEMMA 1. For solutions $u$ and $v$ in Theorem 1, we have

$$
\begin{aligned}
& d\left[u_{n} v\right]=v d u_{n}+u_{n} d v, \\
& d\left[u v_{n}\right]=u d v_{n}+v_{n} d u
\end{aligned}
$$


Proof. Substituting $v(t)=\sum_{k=0}^{\infty} \frac{\eta_{k !}^{k}}{k(k)}$ in the integral form of (4.1a), and using (2.3), we obtain for the right side:

$$
\begin{array}{rl}
\int_{0}^{t} v & d u_{n}+\int_{0}^{t} u_{n} d v \\
= & \int_{0}^{t} \sum_{k=0}^{\infty} \frac{\eta^{k}}{k !} v^{(k)} d u_{n}+\int_{0}^{t} u_{n}\left(q-\lambda_{n}-\eta\right) \sum_{k=0}^{\infty} \frac{\eta^{k}}{k !} u^{(k)} d t+\varepsilon \int_{0}^{t} u_{n} \sum_{k=0}^{\infty} \frac{\eta^{k}}{k !} u^{(k)} d W(t) \\
= & \sum_{k=0}^{\infty} \frac{\eta^{k}}{k !}\left\{\int_{0}^{t} v^{(k)} d u_{n}+\int_{0}^{t} u_{n}\left(q-\lambda_{n}-\eta\right) u^{(k)} d t+\varepsilon \int_{0}^{t} u_{n} u^{(k)} d W(t)\right\}
\end{array}
$$

Since $u_{n}$ is deterministic, $v^{(k)}$ is an $F_{t}$-measurable function satisfying (2.4) for $k \neq 0$, or (2.3) for $k=0$ and $\eta=0$, Ito's formula implies (4.1a) immediately. For (4.1b) we can also prove it in a similar way.

Lemma 2. Suppose that all the assumptions of Theorem 2 are satisfied, and that for $\omega \in \mathscr{C}_{\eta}$, the fixed point of $T(\eta)$ is $\hat{\eta}$, the corresponding solution is $\hat{u}=u(t, \hat{\eta})$. Then

$$
\hat{\eta} \int_{0}^{1} \hat{u} u_{n} d t=\varepsilon \int_{0}^{1} \hat{u} u_{n} d W(t) .
$$

Proof. Multiplying (2.3) by $u_{n}, v_{n}$, we have

$$
\begin{aligned}
v_{n} d u & =\frac{v_{n} v}{p} d t \\
u_{n} d v & =\left(q-\lambda_{n}\right) u u_{n} d t-\eta u u_{n} d t+\varepsilon u u_{n} d W(t), \\
u(0) & =0 \\
v(0) & =v_{0} .
\end{aligned}
$$

By integrating the second equation of (4.3), we obtain

$$
\int_{0}^{1} u_{n} d v=\int_{0}^{1}\left(q-\lambda_{n}\right) u u_{n} d t-\eta \int_{0}^{1} u u_{n} d t+\varepsilon \int_{0}^{1} u u_{n} d W(t)
$$

But from Lemma 1, we know that

$$
d\left(u_{n} v\right)=u_{n} d v+v d u_{n}=u_{n} d v+\frac{v v_{n}}{p} d t,
$$

and then the boundary conditions for $u_{n}$ yield

or

$$
\int_{0}^{1} u_{n} d v+\int_{0}^{1} \frac{v v_{n}}{p} d t=\int_{0}^{1} d\left(u_{n} v\right)=u_{n}(1) v(1)-u_{n}(0) v(0)=0
$$

Therefore (4.1b) and the first equation of (4.3) give the equation

$$
\begin{aligned}
\int_{0}^{1} u_{n} d v & =-\int_{0}^{1} v_{n} d u \\
& =-v_{n}(1) u(1)+v_{n}(0) u(0)+\int_{0}^{1} u d v_{n} \\
& =\int_{0}^{1} u u_{n}\left(q-\lambda_{n}\right) d t-v_{n}(1) u(1) .
\end{aligned}
$$


When $\omega \in \mathscr{C}_{C_{\eta}}$, the contraction mapping theorem implies the existence of the unique fixed point $\hat{\eta}$, and then the corresponding solution $\hat{u}=\sum_{k=0}^{\infty} \frac{\hat{\eta}^{k}}{k !} u^{(k)}$, in which the random function $u^{(k)}$ is independent of $\hat{\eta}$. Setting $\eta=\hat{\eta}$ and denoting $v(t, \hat{\eta})$ by $\hat{v}$, the boundary condition $u(1, \hat{\eta})=0$ reduces the above equation to

$$
\int_{0}^{1} u_{n} d \hat{v}=\int_{0}^{1} \hat{u} u_{n}\left(q-\lambda_{n}\right) d t
$$

and then we can obtain (4.2) by substituting it in (4.4).

Lemma 3. Suppose that the hypotheses of Theorem 2 hold, and that $\chi(\mathscr{A})$ is the indicator of $\mathscr{C}_{C_{\eta}}$. Then there exists a constant $C_{9}$, independent of $\varepsilon$, such that

$$
\left\langle[\hat{\eta} \chi(\mathscr{A})]^{2 n}\right\rangle \leq C_{9} \varepsilon^{2 n}, \quad n=1,2 .
$$

The proof can be obtained in a way very similar to one given by Xia [7], if the initial condition $u(1,0)-\bar{u}(1,0)=u(1,0)-u_{n}(1,0)=u(1,0)$ is noticed.

Lemma 4. Suppose that the hypotheses of Theorem 2 hold. Then there exists a constant $C_{10}$, independent of $\varepsilon$, such that, for $n=1,2$,

$$
\left\langle\left[\frac{\hat{u}-u_{n}}{\varepsilon} \chi(\mathscr{A})\right]\right\rangle^{2 n} \leq C_{10}, \quad\left\langle\left[\frac{\hat{v}-v_{n}}{\varepsilon} \chi(\mathscr{A})\right]^{2 n}\right\rangle \leq C_{10} .
$$

By using Lemma 3, Gronwall's inequality, and equations satisfied by $\hat{u}, \hat{v}, u_{n}, v_{n}$, the proof of this lemma can be derived very easily, and is therefore omitted.

Now we are in a position to give the asymptotic expansion theorem. It is convenient to introduce the following events:

$$
\mathscr{D}_{C_{\eta}}^{\varepsilon}=\left\{\omega|| \int_{0}^{1} \hat{u} u_{n} \chi(\mathscr{A}) d t \mid \geq \frac{1}{2}\right\}, \quad \Omega_{C_{\eta}}^{\varepsilon}=\mathscr{C}_{\eta}^{\varepsilon} \cap \mathscr{D}_{C_{\eta}}^{\varepsilon},
$$

and the approximate solutions:

and

$$
\eta=\left\{\begin{array}{cc}
\varepsilon \int_{0}^{1} u_{n}^{2} d W(t), & \omega \notin \Omega_{C_{\eta}}^{\varepsilon}, \\
\hat{\eta}, & \omega \in \Omega_{C_{\eta}}^{\varepsilon},
\end{array}\right.
$$

$$
u=\left\{\begin{array}{cl}
u_{n}+\varepsilon u^{*}, & \omega \notin \Omega_{C_{\eta}}^{\varepsilon}, \\
\hat{u}, & \omega \in \Omega_{C_{\eta}}^{\varepsilon},
\end{array}\right.
$$

where $u^{*}$ satisfies the initial value problem of the Ito equation:

$$
\begin{aligned}
d u^{*} & =\frac{v^{*}}{p} d t, \\
d v^{*} & =\left[\left(q-\lambda_{n}\right) u^{*}-u_{n} \int_{0}^{1} u_{n}^{2} d W(t)\right] d t+u_{n} d W(t), \\
u^{*}(0) & =0, \\
v^{*}(0) & =0 .
\end{aligned}
$$

Then we have 
THEOREM 3. Let the hypotheses of Theorem 2 be satisfied. Then there exists a constant $C_{\Omega}$, independent of $\varepsilon$, such that

$$
\begin{gathered}
\operatorname{prob}\left\{\Omega_{C_{\eta}}^{\varepsilon}\right\} \geq 1-C_{\Omega} \varepsilon^{2}, \\
\eta=\varepsilon \lambda^{*}(\omega)+O\left(\varepsilon^{2}\right), \\
u=u_{n}+\varepsilon u^{*}+O\left(\varepsilon^{2}\right),
\end{gathered}
$$

where $\lambda^{*}(\omega)=\int_{0}^{1} u_{n}^{2} d w(t)$ is a normal random variable, $u^{*}$ is a Gaussian process with $\left\langle u^{*}\right\rangle=0$, and $O\left(\varepsilon^{2}\right)$ denotes the term with estimation

$$
\sup _{t \in[0,1]}\left\langle\left[\frac{O\left(\varepsilon^{2}\right)}{\varepsilon^{2}}\right]^{2}\right\rangle \leq \text { const. }
$$

Proof. We know that the normal random variable $\int_{0}^{1} u_{n}^{2} d w(t)$ is $F_{1}$-measurable. But the solution of equation (4.10), which is linear, still exists uniquely, and has the matrix expression [12]:

$$
\left(\begin{array}{c}
u^{*} \\
v^{*}
\end{array}\right)=\Phi(t) \int_{0}^{t} \Phi^{-1}(t)\left[\left(\begin{array}{c}
0 \\
-u_{n} \int_{0}^{1} u_{n}^{2} d W(t)
\end{array}\right) d t+\left(\begin{array}{c}
0 \\
u_{n}
\end{array}\right) d W(t)\right] .
$$

Hence

$$
\begin{aligned}
u^{*}(t)=\frac{\varphi_{11}(t)}{v_{0}} & {\left[\int_{0}^{1} u_{n}^{2} d W(t) \int_{0}^{t} u_{n}^{2} d t-\int_{0}^{t} u_{n}^{2} d W(t)\right] } \\
+ & \frac{u_{n}(t)}{v_{0}}\left[-\int_{0}^{1} u_{n}^{2} d W(t) \int_{0}^{t} u_{n} \varphi_{11}(t) d t+\int_{0}^{t} u_{n} \varphi_{11}(t) d W(t)\right] .
\end{aligned}
$$

It is a Gaussian process with mean zero and the second moment:

$$
\begin{aligned}
\left\langle\left[u^{*}\right]^{2}\right\rangle= & \frac{1}{v_{0}^{2}}\left\{\varphi_{11}^{2}(t)\left[\left(\int_{0}^{1} u_{n}^{4} d t\right)\left(\int_{0}^{t} u_{n}^{2} d t\right)^{2}+\int_{0}^{t} u_{n}^{4} d t-2 \int_{0}^{t} u_{n}^{2} d t \int_{0}^{t} u_{n}^{4} d t\right]\right. \\
& +u_{n}^{2}(t)\left[\left(\int_{0}^{1} u_{n}^{4} d t\right)\left(\int_{0}^{t} u_{n} \varphi_{11} d t\right)^{2}\right. \\
& \left.+\int_{0}^{t} u_{n}^{2} \varphi_{11}^{2} d t-2 \int_{0}^{t} u_{n} \varphi_{11} d t \int_{0}^{t} u_{n}^{3} \varphi_{11} d t\right] \\
& +2 \varphi_{11}(t) u_{n}(t)\left[-\left(\int_{0}^{t} u_{n}^{2} d t\right)\left(\int_{0}^{t} u_{n} \varphi_{11} d t\right)\left(\int_{0}^{1} u_{n}^{4} d t\right)+\left(\int_{0}^{t} u_{n}^{2} d t\right)\right. \\
& \left.\left.\cdot\left(\int_{0}^{t} u_{n}^{3} \varphi_{11} d t\right)+\left(\int_{0}^{t} u_{n} \varphi_{11} d t\right)\left(\int_{0}^{t} u_{n}^{4} d t\right)-\int_{0}^{t} u_{n}^{3} \varphi_{11} d t\right]\right\} .
\end{aligned}
$$

Now we consider the event $\mathscr{D}_{C_{\eta}}^{\varepsilon}$. Since

$$
\begin{aligned}
\int_{0}^{1} \hat{u} u_{n} \chi(\mathscr{A}) d t & =\int_{0}^{1}\left[u_{n}+\left(\hat{u}-u_{n}\right)\right] u_{n} \chi(\mathscr{A}) d t \\
& =\left\{\begin{array}{cl}
0, & \omega \notin \mathscr{A}_{C_{\eta}}^{\ell}, \\
1+\int_{0}^{1}\left(\hat{u}-u_{n}\right) u_{n} \chi(\mathscr{A}) d t, & \omega \in \mathscr{A}_{C_{\eta}}^{\varepsilon},
\end{array}\right.
\end{aligned}
$$


for $\omega \notin \mathscr{D}_{C_{\eta}}^{e}$ or $\omega \in\left(\mathscr{D}_{C_{\eta}}^{e}\right)^{\mathrm{C}}$, we have

$$
\begin{aligned}
\operatorname{prob}\left\{\left(\mathscr{D}_{C_{n}}^{e}\right)^{\mathrm{C}}\right\} & =\operatorname{prob}\left\{\left(\mathscr{D}_{C_{n}}^{e}\right)^{\mathrm{C}} \cap\left(\mathscr{C}_{C_{n}}^{e}\right)^{\mathrm{C}}\right\}+\operatorname{prob}\left\{\left(\mathscr{D}_{C_{n}}^{e}\right)^{\mathrm{C}} \cap \mathscr{C}_{C_{n}}^{e}\right\} \\
& \leq \operatorname{prob}\left\{\left(\mathscr{C}_{C_{n}}\right)^{\mathrm{C}}\right\}+\operatorname{prob}\left\{\omega|| 1+\int_{0}^{1}\left(\hat{u}-u_{n}\right) u_{n} \chi(\mathscr{A}) d t \mid<\frac{1}{2}\right\} \\
& \leq C_{A} \varepsilon^{2}+\operatorname{prob}\left\{\omega|| \int_{0}^{1}\left(\hat{u}-u_{n}\right) u_{n} \chi(\mathscr{A}) d t \mid>\frac{1}{2}\right\} .
\end{aligned}
$$

Thus by Lemma 4 , we can find a constant $C_{11}$, independent of $\varepsilon$, such that $\operatorname{Prob}\left\{\left(\mathscr{D}_{C_{n}}^{e}\right)^{\mathrm{C}}\right\} \leq C_{11} \varepsilon^{2}$, and then (4.11a) can be proved immediately.

When $\varepsilon \ll 1, \Omega_{C_{\eta}}^{\varepsilon}$ has large probability. Hence, similar to [7, 8, 9], we can consider (4.8), (4.9) to be the approximations of $\lambda-\lambda_{n}$ and $u$, respectively.

In order to prove (4.11b), we use Lemma 2 , and then find

$$
\frac{\eta-\varepsilon \lambda^{*}}{\varepsilon^{2}}=\left\{\begin{array}{cc}
0, & \omega \notin \Omega_{C_{\eta}}^{\varepsilon} \\
\frac{1}{\varepsilon}\left[\frac{\int_{0}^{1} \hat{u} u_{n} d W(t)}{\int_{0}^{1} \hat{u} u_{n} d t}-\int_{0}^{1} u_{n}^{2} d W(t)\right], & \omega \in \Omega_{C_{\eta}}^{\varepsilon} .
\end{array}\right.
$$

When $\omega \in \Omega_{C_{\eta}}^{e}$, we know that

$$
\begin{aligned}
& \left|\frac{\int_{0}^{1} \hat{u} u_{n} d W(t)}{\int_{0}^{1} \hat{u} u_{n} d t}-\int_{0}^{1} u_{n}^{2} d W(t)\right| \\
& \quad \leq 2\left|\int_{0}^{1} \hat{u} u_{n} d W(t)-\int_{0}^{1} \hat{u} u_{n} d t \int_{0}^{1} u_{n}^{2} d W(t)\right| \\
& \quad=2\left|\int_{0}^{1}\left(\hat{u}-u_{n}\right) u_{n} d W(t)-\int_{0}^{1}\left(\hat{u}-u_{n}\right) u_{n} d t \int_{0}^{1} u_{n}^{2} d W(t)\right| .
\end{aligned}
$$

Since

$$
\frac{\hat{u}-u_{n}}{\varepsilon}=\frac{u^{(0)}-u_{n}}{\varepsilon}+\frac{\hat{\eta}}{\varepsilon} \sum_{k=0}^{\infty} \frac{\hat{\eta}^{k}}{(k+1) !} u^{(k+1)},
$$

we can obtain the estimation for the first term, upon using the Gronwall's inequality to the equation satisfied by $\left(u^{(0)}-u_{n}\right) / \varepsilon$,

$$
\left\langle\sup _{t \in[0,1]}\left(\frac{u^{(0)}-u_{n}}{\varepsilon}\right)^{2}\right\rangle \leq \text { const }
$$

and then the $F_{t}$-measurability of $u^{(0)}$ yields the estimation

$$
\left\langle\left\{\int_{0}^{1}\left[\left(u^{(0)}-u_{n}\right) u_{n} / \varepsilon\right] d W(t)\right\}^{2}\right\rangle \leq \text { const. }
$$


For the integral of the second term we can have

$$
\begin{aligned}
& \left\langle\left(\frac{\hat{\eta}}{\varepsilon}\right)^{2}\left[\sum_{k=0}^{\infty} \frac{\hat{\eta}^{k}}{(k+1) !} \int_{0}^{1} u^{(k+1)} u_{n} d W(t)\right]^{2} \chi(\mathscr{A})\right\rangle \\
& \leq \frac{1}{2}\left[\left\langle\left(\frac{\hat{\eta}}{\varepsilon}\right)^{4} \chi(\mathscr{A})\right\rangle+\left\langle\left[\sum_{k=0}^{\infty} \frac{\hat{\eta}^{k}}{(k+1) !} \int_{0}^{1} u^{(k+1)} u_{n} d W(t)\right]^{4} \chi(\mathscr{A})\right\rangle\right] .
\end{aligned}
$$

But by $(2.5)$ we know that

$$
\left\langle\left[\sum_{k=0}^{\infty} \frac{\hat{\eta}^{k}}{(k+1) !} \int_{0}^{1} u^{(k+1)} u_{n} d W(t)\right]^{4} \chi(\mathscr{A})\right\rangle \leq \text { const. }
$$

Therefore Lemmas 3 and 4 yield the estimations of (4.16), (4.14), and then we have

$$
\left\langle\left[\frac{\eta-\varepsilon \lambda^{*}}{\varepsilon^{2}}\right]^{2}\right\rangle \leq\left\langle\left[\frac{\eta-\varepsilon \lambda^{*}}{\varepsilon^{2}}\right]^{2} \chi(\mathscr{A})\right\rangle \leq \text { const. }
$$

This completes the proof of $(4.11 \mathrm{~b})$. Equation $(4.11 \mathrm{c})$ can be proven in a similar way.

Example. Consider the one-dimensional Schrödinger equation

$$
-u^{\prime \prime}(t)+\varepsilon B(t, \omega) u=\lambda u, \quad u(0)=0, \quad u(1)=0 .
$$

For fixed $n=1,2, \ldots$, since $p(t)=1, q(t)=0$, we have $\lambda_{n}=n^{2} \pi^{2}, u_{n}=\sqrt{2} \sin n \pi t$. It follows that $v_{0}=p(0) u_{n}^{\prime}(0)=\sqrt{2} n \pi$, and then from (3.1) we can obtain

$$
\Phi(t)=\left(\begin{array}{cc}
\cos n \pi t & \frac{1}{n \pi} \sin n \pi t \\
-n \pi \sin n \pi t & \cos n \pi t
\end{array}\right) .
$$

When $\varepsilon$ is small, Theorem 3 gives the approximate eigenvalue and eigenfunction:

$$
\begin{aligned}
\lambda= & \lambda_{n}+\varepsilon \lambda^{*}(\omega)+O\left(\varepsilon^{2}\right)=\lambda_{n}+2 \varepsilon \int_{0}^{1} \sin ^{2} n \pi t d W(t)+O\left(\varepsilon^{2}\right), \\
u= & u_{n}+\varepsilon u^{*}+O\left(\varepsilon^{2}\right) \\
= & \sqrt{2} \sin n \pi t+\sqrt{2} \varepsilon\left\{\left(\frac{\sin n \pi t}{n \pi}-t \cos n \pi t\right)\right. \\
& \left.\cdot \int_{0}^{1} W(x) \sin 2 n \pi x d x+\int_{0}^{t} W(x) \sin (2 n \pi x-n \pi t) d x\right\}+O\left(\varepsilon^{2}\right),
\end{aligned}
$$

where $\lambda^{*}$ is a normal random variable with expectation zero and covariance $3 / 2$, and $u^{*}$ is a Gaussian process with expectation zero.

\section{REFERENCES}

[1] W. E. Boyce, Random eigenvalue problems, in Probabilistic Methods in Applied Mathematics, Vol. 1, A. T. Bharucha-Reid (Editor), Academic Press, New York, 1968, 1-73

[2] J. vom Scheidt and W. Purkert, Random Eigenvalue Problems, Akademie-Verlag, Berlin, 1983

[3] W. E. Boyce and Ning-Mao Xia, The approach to normality of the solutions of random boundary and eigenvalue problems with weakly correlated coefficients, Quart. Appl. Math. 40, 419-445 (1983) 
[4] W. E. Boyce and Ning-Mao Xia, Upper bounds for the means of eigenvalues of random boundary value problems with weakly correlated coefficients, Quart. Appl. Math. 42, 439-454 (1985)

[5] G. Birkhoff and G.-C. Rota, Ordinary Differential Equations, 3rd ed., Wiley, New York, 1978

[6] Ning-Mao Xia, The distribution function of the solution of the random eigenvalue problem for differential equations, J. Math. Anal. Appl. 130, 577-589 (1988)

[7] Ning-Mao Xia, The solutions for the two-point boundary value problems of stochastic differential equations containing small white noises, Acta Mathematicae Applicate 8, 340-350 (1985) (in Chinese)

[8] Ning-Mao Xia, The density function of the solution of a two-point boundary value problem containing small stochastic processes, Quart. Appl. Math. 46, 29-47 (1988)

[9] Ning-Mao Xia, Two-point boundary value problem of linear random differential equations containing small parameter and applications to one-dimensional Helmholtz equation, Journal of Systems Science and Mathematical Sciences 7, 129-137 (1987) (in Chinese)

[10] Ning-Mao Xia, Upper bounds for the means of the higher eigenvalues of random eigenvalue problems, Journal of East China Institute of Chemical Technology 12, 115-119 (1986) (in Chinese)

[11] I. I. Gikhman and A. V. Skorokhod, Stochastic Differential Equations, Springer-Verlag, New York, Heidelberg, Berlin, 1972

[12] L. Arnold, Stochastic Differential Equations: Theory and Applications, John-Wiley, New York, 1974

[13] B. S. White and J. N. Franklin, A Limit Theorem for Stochastic Two-point Boundary Value Problems of Ordinary Differential Equations, Commun. Pure Appl. Math. 32, 253-275 (1979)

[14] L. A. Pastur, Spectra of random self-adjoint operators, Russian Math. Surveys 28, 1-67 (1973) 